\title{
Fetal hyperglycemia and a high-fat diet contribute to aberrant glucose tolerance and hematopoiesis in adult rats
}

\author{
Emily K. Blue ${ }^{1,2}$, Kimberly Ballman ${ }^{1,2}$, Frances Boyle 1,2 , Eunjin Oh ${ }^{1,2}$, Tatsuyoshi Kono ${ }^{1,2}$, Sara K. Quinney ${ }^{3}$,
} Debbie C. Thurmond ${ }^{1,2,4,5}$, Carmella Evans-Molina ${ }^{1,2,4-6}$ and Laura S. Haneline ${ }^{1,2,4,7,8}$

\begin{abstract}
BACKGROUND: Children exposed to gestational diabetes mellitus (GDM) during pregnancy are at increased risk of obesity, diabetes, and hypertension. Our goal was to identify metabolic and hematopoietic alterations after intrauterine exposure to maternal hyperglycemia that may contribute to the pathogenesis of chronic morbidities.
\end{abstract}

METHODS: Streptozotocin treatment induced maternal hyperglycemia during the last third of gestation in rat dams. Offspring of control mothers (OCM) and diabetic mothers (ODM) were evaluated for weight, glucose tolerance, insulin tolerance, and hematopoiesis defects. The effects of aging were examined in normal and high-fat diet (HFD)-fed young (8-wk-old) and aged (11-mo-old) OCM and ODM rats.

RESULTS: Young adult ODM males on a normal diet, but not females, displayed improved glucose tolerance due to increased insulin levels. Aged ODM males and females gained more weight than OCM on a HFD and had worse glucose tolerance. Aged ODM males fed a HFD were also neutrophilic. Increases in bone marrow cellularity and myeloid progenitors preceded neutrophilia in ODM males fed a HFD.

CONCLUSION: When combined with other risk factors like HFD and aging, changes in glucose metabolism and hematopoiesis may contribute to the increased risk of obesity, type 2 diabetes, and hypertension observed in children of GDM mothers.

G estational diabetes mellitus (GDM) most often occurs during the third trimester of pregnancy when maternal insulin resistance increases and insulin secretion is inadequate, leading to hyperglycemia (1). Between $5-10 \%$ of pregnancies in the United States are affected by GDM (2), which doubled from 1994 to $2000(1,3)$. GDM is linked to many health complications in both the mother and fetus, including preeclampsia, fetal macrosomia, and fetal death (4). In addition to these acute complications, GDM exposure in utero increases the child's risk of disease later in life. Children of mothers with GDM are more susceptible to obesity, type 2 diabetes, hypertension, and the metabolic syndrome (5-8). However, the cellular and molecular mechanisms that underlie the link between prenatal exposure to diabetes and disease later in life are not well understood.

Since glucose freely crosses the placenta to the fetal circulation, manipulation of maternal glycemia is a strategy commonly used to model diabetes in pregnancy in animals. The majority of previous studies focused on pregestational diabetic models. These studies demonstrate that intrauterine exposure to hyperglycemia leads to altered glucose tolerance $(9,10)$, bone development $(11,12)$, renal function $(11,13)$, and vascular function $(9,14)$. However, few studies have been conducted that replicate the pathologic effects of GDM with onset of hyperglycemia during late pregnancy (15-19).

There are a variety of environmental factors such as diet, exercise, and aging that may influence the development of obesity, type 2 diabetes, and hypertension. In addition to intrauterine exposure to GDM, consumption of a high-fat diet (HFD) is a risk factor for these chronic morbidities. Moreover, these diseases are common in older populations, leading to the supposition that aging also promotes disease pathogenesis $(20,21)$. Furthermore, many inflammatory cell types, including macrophages and neutrophils, contribute to obesity-linked systemic inflammation (22-24). We hypothesize that the combination of prenatal exposure to hyperglycemia, postnatal consumption of a HFD, and aging synergistically increase the risk of obesity and diabetes, partly due to the contributions of changes in $\beta$-cell function and in hematopoiesis. To test this hypothesis, we used an established rat model of GDM in which hyperglycemia occurs during the last third of gestation. Previous studies using this model demonstrated sex differences in metabolic and vascular phenotypes (16-18). Therefore, we prospectively examined males and females for all phenotypes tested. Young and aged offspring from these pregnancies were challenged with a HFD. In addition to evaluating for effects on weight gain and glucose tolerance, studies were conducted to examine

\footnotetext{
'Department of Pediatrics, Indiana University School of Medicine, Indianapolis, Indiana; ${ }^{2}$ Herman B Wells Center for Pediatric Research, Indiana University School of Medicine, Indianapolis, Indiana; ${ }^{3}$ Department of Obstetrics and Gynecology, Indiana University School of Medicine, Indianapolis, Indiana; ${ }^{4}$ Department of Cellular \& Integrative Physiology, Indiana University School of Medicine, Indianapolis, Indiana; ${ }^{5}$ Department of Biochemistry and Molecular Biology, Indiana University School of Medicine, Indianapolis, Indiana; ${ }^{6}$ Department of Medicine, Indiana University School of Medicine, Indianapolis, Indiana; ${ }^{7}$ Department of Microbiology \& Immunology, Indiana University School of Medicine, Indianapolis, Indiana; ${ }^{8}$ Indiana University Simon Cancer Center, Indiana University School of Medicine, Indianapolis, Indiana. Correspondence: Laura S. Haneline (Ihanelin@iu.edu)
} 
whether alterations in circulating inflammatory cell levels and hematopoiesis existed.

\section{RESULTS}

Streptozotocin (STZ) induces necrotic death of rat pancreatic $\beta$ cells resulting in insulin deficiency and subsequent hyperglycemia (25). To induce hyperglycemia in late gestation to model human GDM, pregnant rat dams were injected with either citrate buffer (control mothers) or STZ (GDM mothers, $45 \mathrm{mg} / \mathrm{kg} \mathrm{STZ}$ ) on day 12 of gestation, similar to previous studies (16-19). Injection of STZ induced a rapid increase in maternal blood glucose levels by gestational day 15 (Figure 1a). To confirm a loss of functional $\beta$ cells in GDM dams, pancreata were evaluated for insulin expressing $\beta$ cells. As expected, STZ-treated dams had reduced $\beta$ cell area (Figure $1 b$, control dams and Figure 1c, GDM dams). To assess for potential direct effects of STZ on fetal $\beta$ cells, quantitation of pancreatic $\beta$ cell area was performed. In contrast to data in the mothers, no differences were observed in $\beta$ cell mass between offspring of control mothers (OCM) and offspring of diabetic mothers (ODM) neonates (Figure 1d,e). Quantitative analysis of the insulin-positive $\beta$ cell areas revealed similar $\beta$ cell islet areas for OCM and ODM $(3.4 \pm 2.0 \%$ for OCM and $3.0 \pm 2.1 \%$ for ODM; $P=0.82$ ). Dam weight gain was lower in GDM mothers compared to controls (Table 1). There were no differences in litter size, but an increase in maternal deaths during labor was observed in the GDM mothers $(P=0.009$, Table 1$)$. On postnatal day 1 , pups from citrate- and STZ-injected dams were crossfostered to mothers who had not been subjected to STZ or citrate injection during pregnancy. The OCM and ODM were evaluated for weight gain, glucose tolerance, insulin tolerance, and hematopoiesis.

\section{Maternal Hyperglycemia Causes Hypoglycemia in Male ODM}

Exposure to maternal hyperglycemia did not alter the birth weights of surviving males (OCM $7.1 \pm 0.7 \mathrm{~g}, n=63$; ODM $6.9 \pm 0.9 \mathrm{~g}, n=54$ ) or females (OCM $6.7 \pm 0.7 \mathrm{~g}, n=64$; ODM $6.6 \pm 0.8 \mathrm{~g}, n=54)$. To evaluate for neonatal hypoglycemia and hyperinsulinemia, blood glucose and plasma insulin levels were measured in OCM and ODM on postnatal day 1 in random-fed neonates. Male ODM had significantly decreased blood glucose levels compared to male OCM $(75 \pm 10 \mathrm{mg} / \mathrm{dl}$, $n=9$ vs. $87 \pm 15 \mathrm{mg} / \mathrm{dl}, n=8 ; P=0.016$ ). Plasma insulin levels in males did not reach statistical significance, but trended higher in male ODM (male OCM $0.6 \pm 0.2 \mathrm{ng} / \mathrm{ml}, n=8$; ODM $0.9 \pm 0.4 \mathrm{ng} / \mathrm{ml}, n=10 ; P=0.09$ ). Blood glucose levels were also lower in female ODM compared to OCM $(69 \pm 9 \mathrm{mg} / \mathrm{dl}, n=12$ vs. $75 \pm 6 \mathrm{mg} / \mathrm{dl}, n=9 ; P=0.049)$. Insulin levels in female ODM were not statistically different from female OCM $(0.8 \pm 0.7 \mathrm{ng} /$ $\mathrm{ml}, n=9$ vs. $0.5 \pm 0.3 \mathrm{ng} / \mathrm{ml}, n=9 ; P=0.33$ ).

\section{Young Male ODM, but Not Female ODM, Have Increased Glucose Tolerance}

In humans, children of mothers with GDM have an increased risk of obesity, insulin resistance, and the metabolic syndrome at young ages (5-8). To determine if hyperglycemia during late gestation in rats led to any of these metabolic changes, weekly weight checks and monthly glucose tolerance tests were conducted in young (3-13 wk old) OCM and ODM. No differences in weight were detected in OCM and ODM rats during this time. Interestingly, a small but significant increase in glucose tolerance was seen in young male ODM compared to male OCM (Figure 2a,c). In contrast, there were no differences in glucose tolerance between OCM and ODM females (Figure 2b,c). Similar results for glucose tolerance were observed at 4 and 13 wk of age (data not shown).
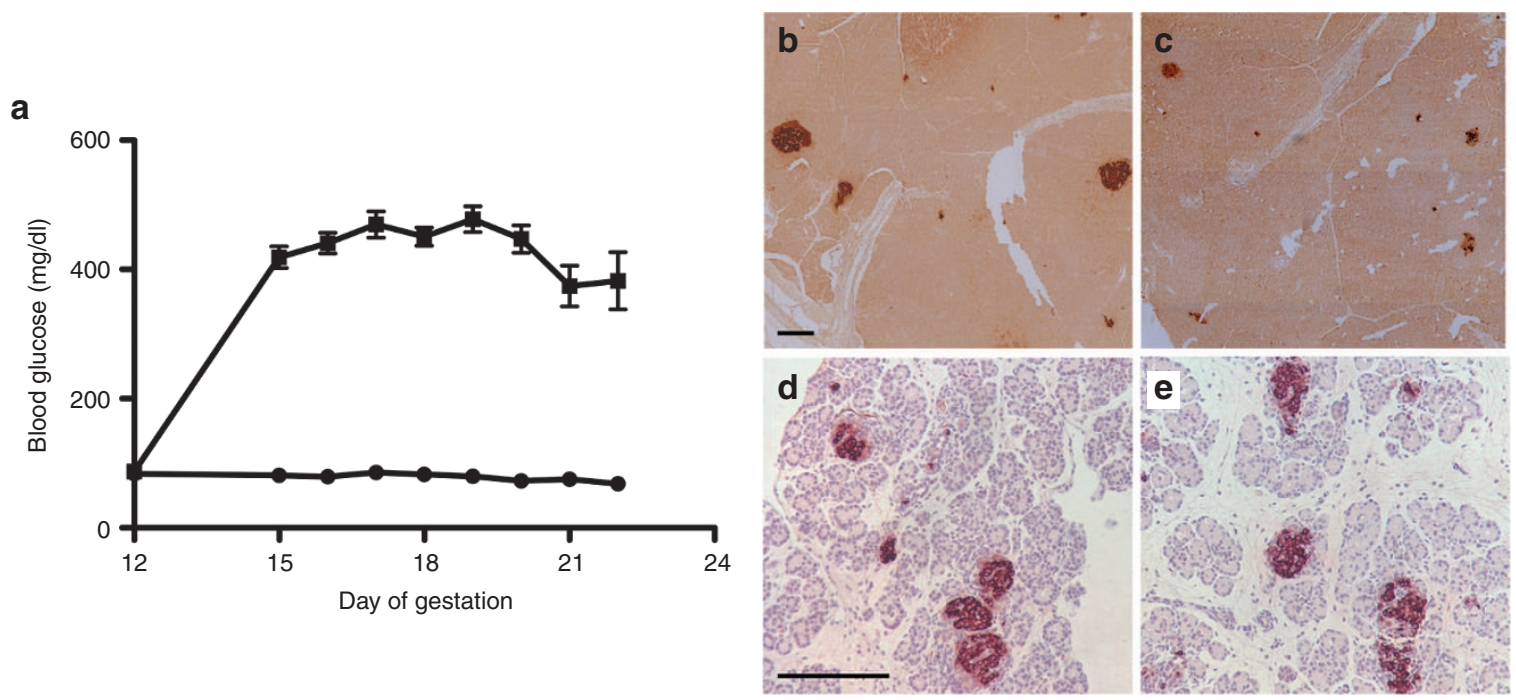

Figure 1. Streptozotocin (STZ) induced elevated glucose levels during the last third of gestation, due to $\beta$ cell death. (a) After either injection of citrate (control, circles) or STZ (gestational diabetes mellitus (GDM), squares) at gestational day 12, morning blood glucose levels were tracked in the timed pregnant mothers. Graph shows mean and SEM glucose levels. (b-e) Immunohistochemical measurement of pancreatic $\beta$ cells by insulin staining reveals loss of $\beta$ cells in dams, but not for neonatal pups. Representative images are shown for (b) control dams, (c) GDM dams, (d) offspring of control mothers neonates, and (e) offspring of diabetic mothers neonates. Line denotes scale for $200 \mu \mathrm{m}$. 


\section{Articles | Blue et al.}

Enhanced glucose tolerance can be attributed to either increased insulin secretion or improved insulin sensitivity of peripheral tissues. To examine for evidence of increased insulin secretion, insulin levels were measured following glucose injection during a glucose tolerance test. These studies showed that ODM males have increased plasma insulin levels in response to glucose injection compared to OCM males (Figure 2d). Consistent with the findings for glucose tolerance, insulin levels in female OCM and ODM were similar (Figure 2e). To evaluate males for changes in insulin sensitivity, insulin tolerance tests were performed. OCM and ODM males had similar insulin sensitivity (Figure 2f). Collectively, these

Table 1. Maternal data for rat GDM model

\begin{tabular}{lccc}
\hline & $\begin{array}{c}\text { Control } \\
\text { mother }\end{array}$ & $\begin{array}{c}\text { GDM } \\
\text { mother }\end{array}$ & \\
\cline { 2 - 3 } Total number per group & 20 & 30 & P value \\
\hline Weight: GD 12 (g) & $257 \pm 22$ & $262 \pm 16$ & 0.57 \\
Weight: GD 21 (g) & $346 \pm 34$ & $329 \pm 31$ & 0.21 \\
Maternal weight gain (g) & $91 \pm 15$ & $68 \pm 21^{*}$ & 0.03 \\
Litter size (live pups) & $10.7 \pm 2.1$ & $10.7 \pm 4.1$ & 0.99 \\
Maternal deaths during labor, $n(\%)$ & $1(5 \%)$ & $5(17 \%)^{* *}$ & 0.009 \\
\hline
\end{tabular}

Means \pm SD are shown.

GDM, gestational diabetes mellitus.

${ }^{*} P<0.05$ by unpaired $t$-test. ${ }^{*} P<0.01$ by $X^{2}$ test. data suggest that the improved glucose tolerance observed in the young male ODM is likely due to an aberrant increase in insulin secretion following glucose administration.

\section{Consumption of a HFD Diminishes Differences in Glucose Tolerance in Young Male ODM}

Unexpectedly, young male ODM displayed increased glucose tolerance (Figure 2a). These rats were fed a normal diet with low fat content ( $14 \% \mathrm{kcal}$ from fat). Since increased caloric intake is linked to weight gain, obesity, and risk of type 2 diabetes, we hypothesized that the dietary stress of a HFD would lead to increased weight and impaired glucose tolerance, especially in the ODM rats. To test this hypothesis, a cohort of OCM and ODM were weaned from their mothers to either the normal diet or a HFD ( $60 \% \mathrm{kcal}$ from fat). Rats were weighed weekly to track weight changes (Figure 3a,b). Consumption of a HFD over the 23-wk feeding period resulted in a significant weight gain in males $(519 \pm 34$ vs. $441 \pm 31 \mathrm{~g}, P<0.0001)$ and females $(258 \pm 32 \mathrm{~g}$ vs. $231 \pm 19 \mathrm{~g}, P=0.004)$, revealing significant effects of HFD on weight gain $(P<0.0001$ by two-way ANOVA). However, no differences were detected between OCM and ODM.

Glucose tolerance tests were performed on 8-wk-old rats to determine if ODM rats on a HFD had impaired glucose tolerance. Similar to data illustrated in Figure 2a, the ODM males fed a normal diet showed slightly improved glucose tolerance compared to OCM controls (Figure 3c). Interestingly,
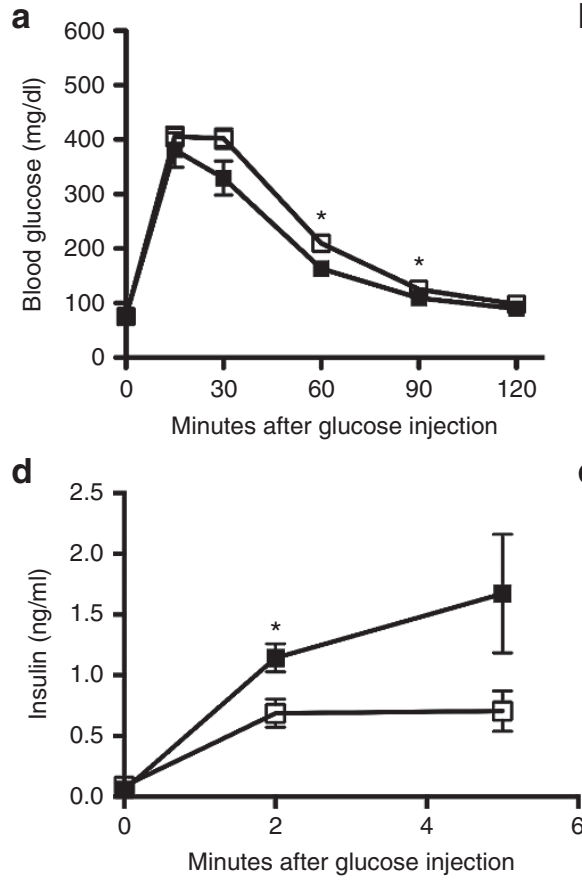

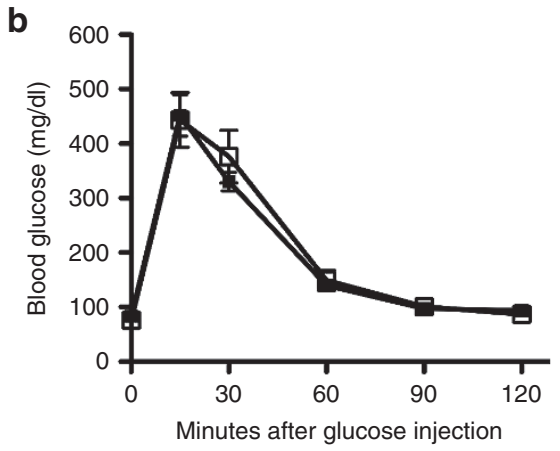

e

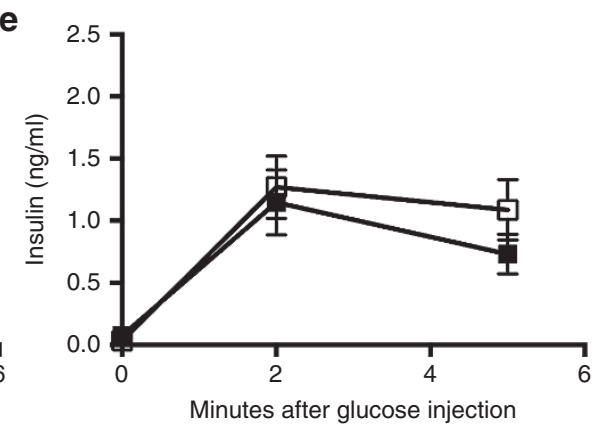

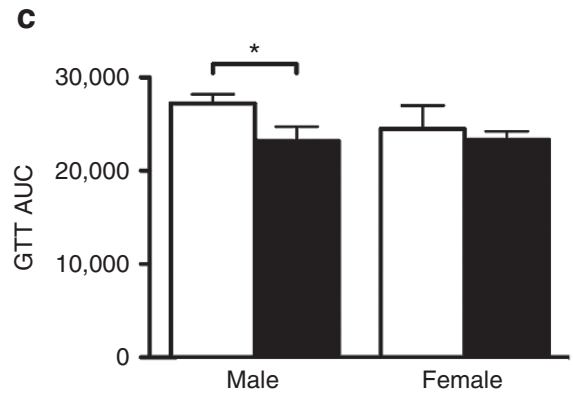

f

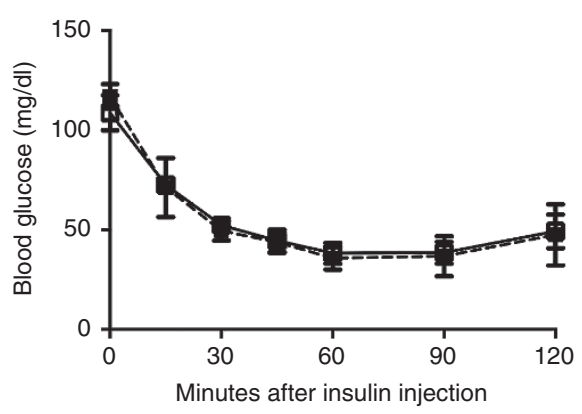

Figure 2. Eight-week-old male offspring of diabetic mothers (ODM) have improved glucose tolerance due to increased insulin response to hyperglycemia. For all graphs, offspring of control mothers (OCM) is shown in white, and ODM is in black. (a,b) A GTT was administered to fasted 8-wk-old (a) male and (b) female rats. ${ }^{*} P<0.05$ by repeated measures ANOVA followed by Sidak's multiple comparisons. (c) Area under the curve measurements for GTT are shown. Significant effects of ODM were observed by two-way ANOVA. ${ }^{*} P<0.05$ by Tukey's multiple comparisons. OCM: open bars; ODM: closed bars. For panels $(\mathbf{a}-\mathbf{c}) n=8$ males and $n=7$ females. (d,e) Insulin levels were quantified in a separate cohort of OCM and ODM within 5 min after glucose injection. Graphs show plasma insulin levels before and after glucose injection for (d) males (OCM, $n=8$; ODM, $n=8)$ and (e) females (OCM, $n=7$; ODM, $n=9$ ). ${ }^{*} P<0.05$ by repeated measures ANOVA followed by Sidak's multiple comparisons. (f) Insulin tolerance tests were performed on 8 -wk-old male OCM and ODM. Graph shows glucose levels at various times after injection of insulin ( $n=4$ OCM and ODM). No significant differences in insulin tolerance were detected using repeated measures ANOVA. Graphs show mean and SEM values. GTT, glucose tolerance test. 

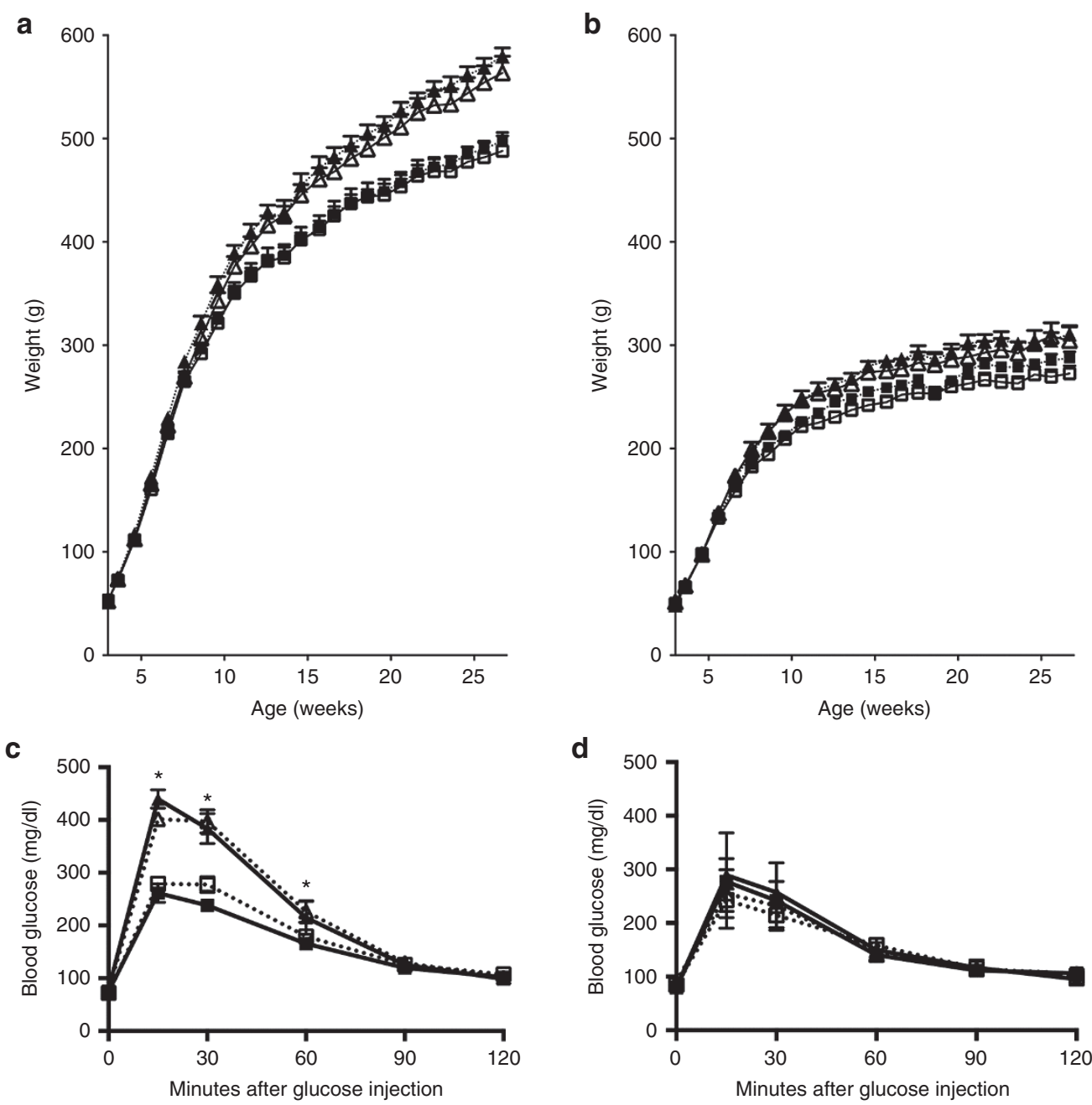

Figure 3. High-fat diet (HFD) induces weight gain in offspring of control mothers (OCM) and offspring of diabetic mothers (ODM) males and diminishes differences in glucose tolerance in young males. After weaning, young males were fed either a normal diet or a HFD. For (a-d), OCM is shown in white symbols, ODM shown in black symbols, normal diet shown in squares, and HFD shown in triangles. (a,b) Weights are shown for OCM and ODM (a) males and (b) females. Both OCM and ODM males and females fed a HFD show increased body weight compared to those fed a normal diet starting at age $10 \mathrm{wk}$ for males and $11 \mathrm{wk}$ for females $(P<0.05$ by repeated measures two-way ANOVA followed by Tukey's multiple comparisons). However, there was no significant difference in weight at these ages between OCM and ODM on either diet for either males or females. For males, OCM normal, $n=9$; ODM normal, $n=9$; OCM HFD, $n=8$; ODM HFD, $n=8$. For females, OCM normal, $n=9$; ODM normal, $n=9$; OCM HFD, $n=9$; ODM HFD, $n=10$. (c,d) GTT results for fasted 8-wk-old OCM and ODM (c) males and (d) females. Males fed a HFD show impaired glucose tolerance $(* P<0.05$ for HFD effect by repeated measures two-way ANOVA with Tukey's multiple comparisons). Females showed no effect of HFD on glucose tolerance. There was no significant difference in glucose tolerance between 8-wk-old OCM and ODM on a HFD. For males, OCM normal, $n=5$; ODM normal, $n=5$; OCM HFD, $n=5$; ODM HFD, $n=4$. For females, $n=5$ for all groups. Graphs show mean and SEM values. GTT, glucose tolerance test.

Table 2. Weight gain over 2 mo in 11-mo-old rats

\begin{tabular}{|c|c|c|c|c|c|c|c|c|}
\hline & \multicolumn{4}{|c|}{ Males } & \multicolumn{4}{|c|}{ Females } \\
\hline & \multicolumn{2}{|c|}{ Normal } & \multicolumn{2}{|r|}{ HFD } & \multicolumn{2}{|c|}{ Normal } & \multicolumn{2}{|c|}{ HFD } \\
\hline & $\mathrm{OCM}, n=5$ & $\mathrm{ODM}, n=5$ & $\mathrm{OCM}, n=5$ & $\mathrm{ODM}, n=5$ & OCM, $n=6$ & $\mathrm{ODM}, n=5$ & OCM, $n=6$ & ODM,$n=6$ \\
\hline
\end{tabular}

Means \pm SD are shown. Statistical significance defined as $P<0.05$ by two-way ANOVA followed by Sidak's multiple comparisons.

HFD, high-fat diet; OCM, offspring of control mothers; ODM, offspring of diabetic mothers.

${ }^{*} P<0.05$ compared to OCM males on same diet. ${ }^{*} P<0.05$ compared to males on normal diet. ${ }^{\dagger} P<0.05$ compared to ODM females on normal diet.

consumption of a HFD led to reduced glucose tolerance in both OCM and ODM males, eliminating the improvement in glucose tolerance observed in the ODM males on a normal diet (Figure 3c). In contrast, HFD did not impact glucose tolerance in young females (Figure 3d). Thus, HFD consumption eliminated improvements in glucose tolerance of young ODM males. However, HFD induced similar glucose intolerance in young OCM and ODM males. 


\section{Articles | Blue et al.}

\section{Aged ODM Have Increased Susceptibility to Metabolic Derangements of HFD}

We hypothesized that postnatal exposure to the combination of environmental stresses seen clinically (i.e., aging and HFD) would lead to profound impairments in the glucose tolerance of offspring exposed to intrauterine hyperglycemia. To test this hypothesis, 9-mo-old OCM and ODM were
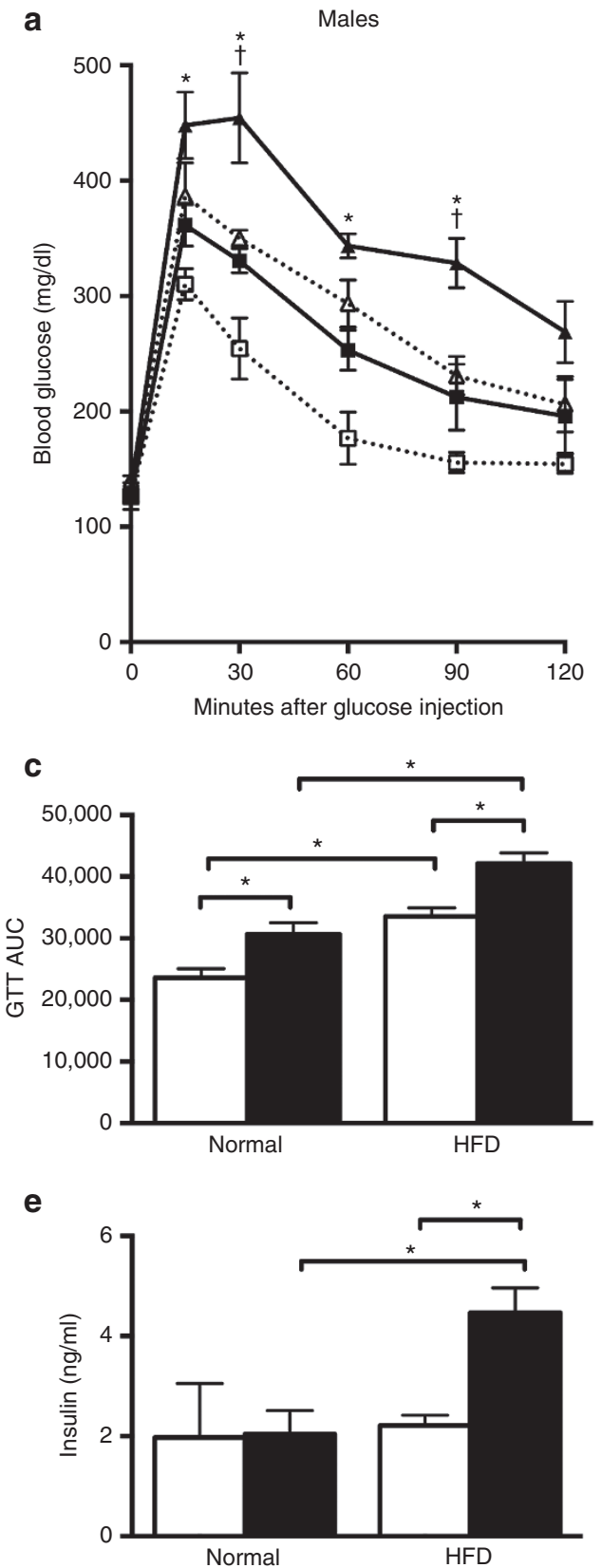

fed either a normal diet or a HFD for 2 mo. This approach was chosen so that adult rats were fed a HFD for a similar length of time as studies in younger rats and to avoid potential developmental consequences of HFD consumption from the time of weaning. Consumption of a HFD for 2 mo by older males led to increased weight gain in both OCM and ODM males (Table 2). However, the male ODM HFD group

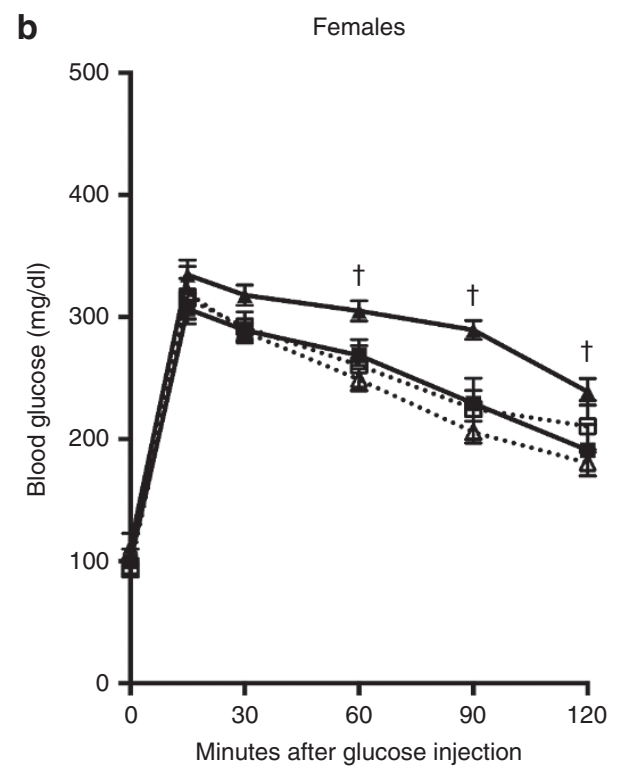

d

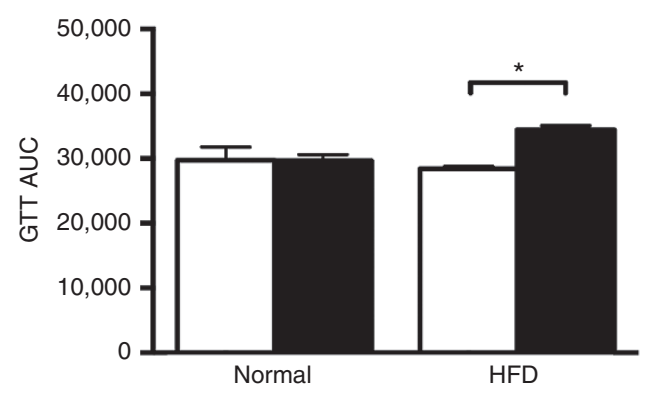

f

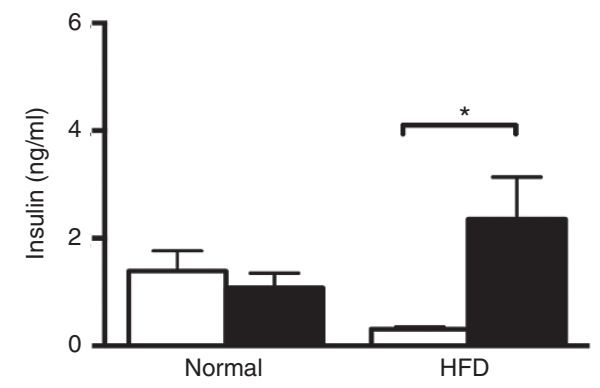

Figure 4. High-fat diet (HFD) increases weight gain in offspring of diabetic mothers (ODM) males and females and impairs glucose tolerance. Ninemonth-old offspring of control mothers (OCM) and ODM males and females were fed either normal diet or HFD for 2 mo until the age of 11 mo. OCM: open bars; ODM: closed bars. (a,b) GTT results from (a) male and (b) female 11-mo-old OCM and ODM rats. OCM are shown in white symbols, ODM shown in black symbols, normal diet shown in squares, and HFD in triangles. ${ }^{\dagger} P<0.05$ for significant effect of ODM and ${ }^{*} P<0.05$ for significant effect of HFD by repeated measures ANOVA with Tukey's multiple comparisons. (c,d) Area under the curve measurements also showed significant effects of both ODM and HFD for the males and ODM and the interaction between ODM and HFD for the females. For (a,c) males, OCM normal $n=4$, ODM normal $n=5$, OCM HFD $n=6$, ODM HFD $n=5$. For (b,d) females, OCM normal $n=6$, ODM normal $n=5$, OCM HFD $n=5$, ODM HFD $n=6$. (e,f) Random-fed insulin measurements are shown for 11-mo-old (e) males and (f) females. ${ }^{*} P<0.05$ by two-way ANOVA with Tukey's multiple comparisons. For (e) males, OCM normal $n=3$, ODM normal $n=5$, OCM HFD $n=5$, ODM HFD $n=5$. For (f) females, OCM normal $n=5$, ODM normal $n=5$, OCM HFD $n=6$, ODM HFD $n=6$. Graphs show mean and SEM values. GTT, glucose tolerance test. 
a
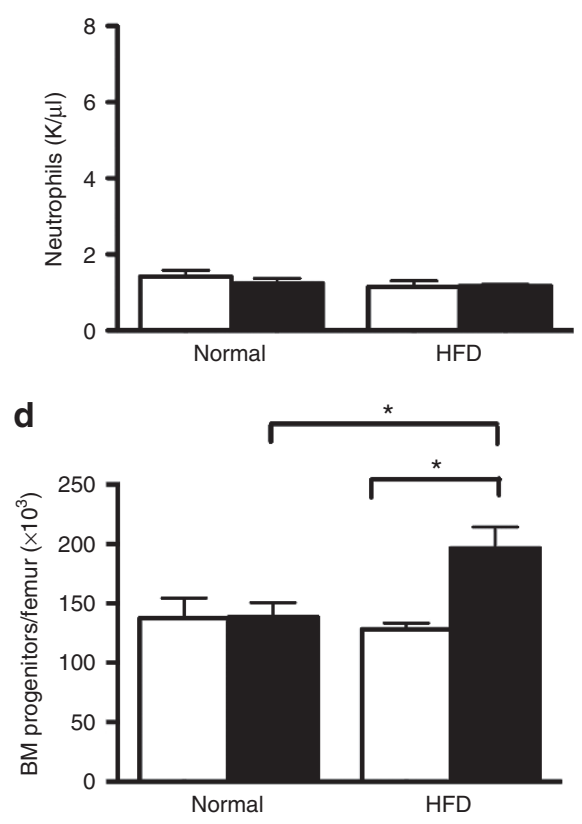

g

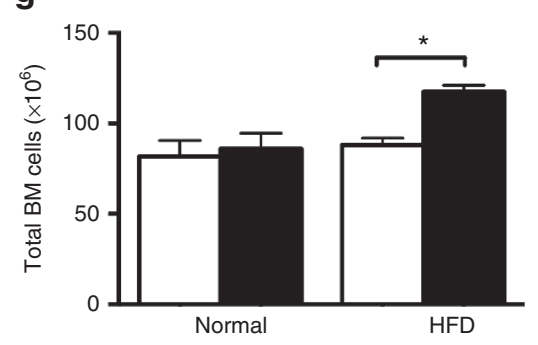

b

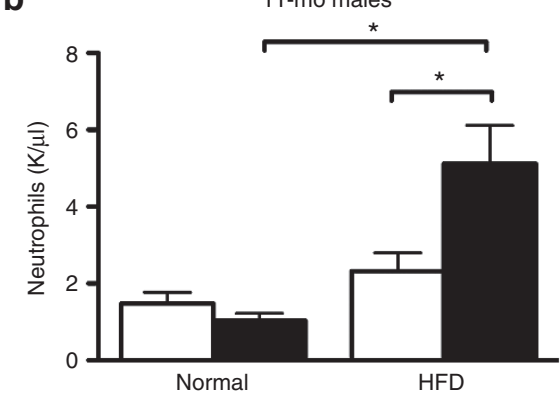

e

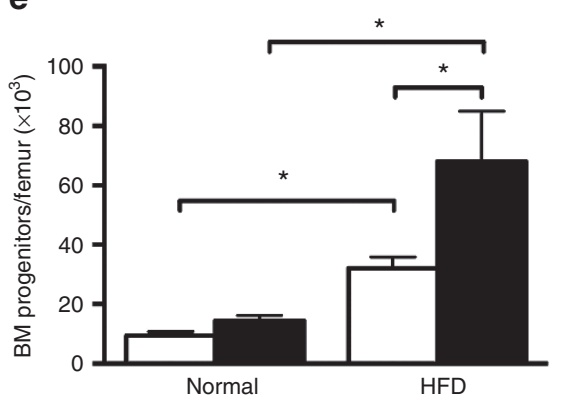

h

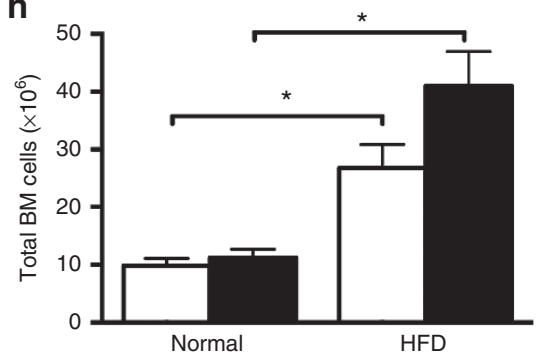

C

11-mo females

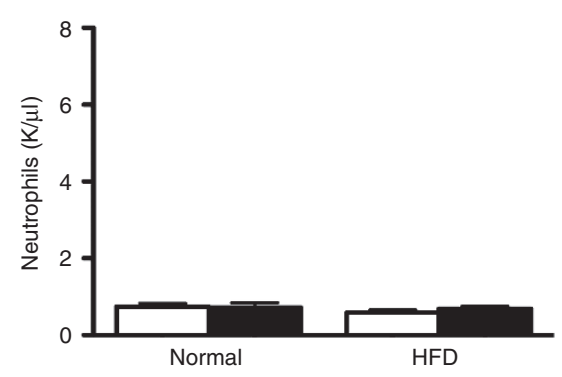

f

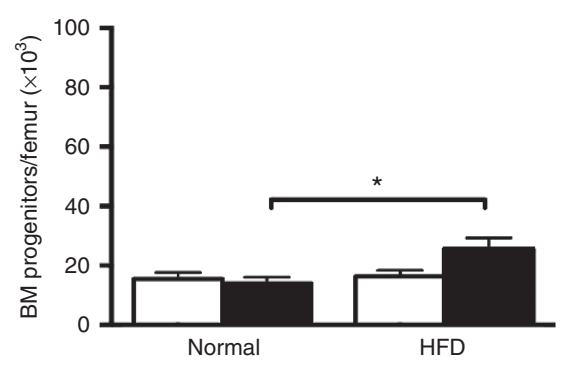

i

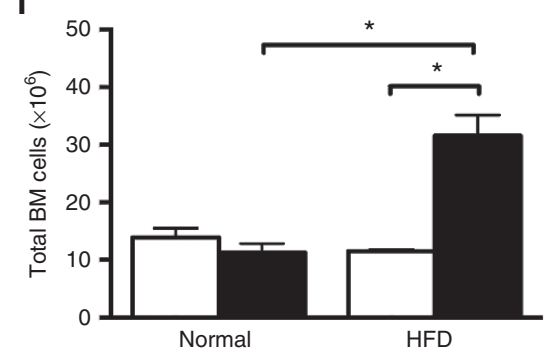

Figure 5. Male offspring of diabetic mothers (ODM) fed a high-fat diet (HFD) have neutrophilia and increased bone marrow (BM) progenitor cells. Neutrophil counts, bone marrow myeloid progenitors, and total bone marrow cellularity measurements were performed on the same animals at each age. Offspring of control mothers (OCM): open bars; ODM: closed bars. (a-c) Neutrophils (K/ $\mu$ l) were quantified by complete blood cell count (a) in males at $6 \mathrm{mo}$, (b) in males at $11 \mathrm{mo}$, and (c) in females at $11 \mathrm{mo}$ of age. Eleven-month-old ODM males fed a HFD exhibited neutrophilia (b), while all other experimental groups had normal neutrophil levels. For (a) males at $6 \mathrm{mo}, n=8$ for all groups. For (b) males at $11 \mathrm{mo}$, OCM normal $n=3$, ODM normal $n=5$, OCM HFD $n=5$, ODM HFD $n=5$. For (c) females at 11 mo, OCM normal $n=5$, ODM normal $n=5$, OCM HFD $n=6$, ODM HFD $n=6$. (d-f) Myeloid progenitors were quantified per femur in (d) 6-mo-old males, (e) 11-mo-old males, and (f) 11-mo-old females. All HFD-fed ODM males, and 11-mo-old HFD-fed ODM females had increased myeloid progenitors. For (d) males at 6 mo, OCM normal $n=8$, ODM normal $n=8$, OCM HFD $n=7$, ODM HFD $n=7$. For (e) males at $11 \mathrm{mo}, n=5$ for all four groups. For (f) females at 11 mo, OCM normal $n=5$, ODM normal $n=5$, OCM HFD $n=5$, ODM HFD $n=4$. (g-i) Total bone marrow cellularity was measured in (g) 6-mo-old males, (h) 11-mo-old males, and (i) 11-mo-old females. Bone marrow cellularity was increased in HFD-fed ODM males and females. ${ }^{*} P<0.05$ by two-way ANOVA with Tukey's multiple comparisons. For (g) males at 6 mo, OCM normal $n=8$, ODM normal $n=8$, OCM HFD $n=6$, ODM HFD $n=6$. For (h) males and (i) females at $11 \mathrm{mo}, n=5$ for each group. Graphs show mean and SEM values.

gained significantly more weight compared to the OCM fed a HFD. For female rats, only the ODM on a HFD for 2 mo exhibited significant weight gain (Table 2). Thus, in both males and females, the combination of intrauterine exposure to hyperglycemia, HFD consumption, and aging induced the greatest weight gains.

We next examined the effect of the postnatal stress on glucose tolerance of aged OCM and ODM rats. Even on a normal diet, 11-mo-old ODM males exhibited impaired glucose tolerance when compared with OCM males (Figure 4a,c). While HFD consumption impaired glucose tolerance in both OCM and ODM males, ODM HFD-fed males displayed the worst glucose tolerance. For the females, impaired glucose tolerance was only detected in the 11-mo-old aged ODM HFD-fed group (Figure $\mathbf{4 b}, \mathbf{d}$ ). Thus, in both males and females, the experimental group with the worst glucose tolerance was the 11-mo-old, HFD-fed ODM. Insulin measurements from male and female rats showed elevated insulin in ODM HFD-fed males and females (Figure 4e,f). These data indicate that prenatal and postnatal factors contribute to the development of insulin resistance and glucose intolerance.

\section{HFD-Fed ODM Males Exhibit Neutrophilia and Increased Bone Marrow Myeloid Progenitors}

To determine if there were differences in circulating leukocytes in OCM and ODM rats fed a HFD, complete blood cell counts were performed. No differences in circulating leukocytes were detected in 6-mo-old rats (Figure 5a). However, an increase 


\section{Articles | Blue et al.}

Table 3. Adipokine levels in 11-mo-old rats

\begin{tabular}{|c|c|c|c|c|c|c|c|c|}
\hline & \multicolumn{4}{|c|}{ Males } & \multicolumn{4}{|c|}{ Females } \\
\hline & \multicolumn{2}{|c|}{ Normal } & \multicolumn{2}{|r|}{ HFD } & \multicolumn{2}{|c|}{ Normal } & \multicolumn{2}{|c|}{ HFD } \\
\hline & $\mathrm{OCM}, n=3$ & ODM, $n=4$ & $\mathrm{OCM}, n=5$ & ODM, $n=5$ & $\mathrm{OCM}, n=4$ & ODM, $n=4$ & $\mathrm{OCM}, n=6$ & ODM,$n=6$ \\
\hline $\begin{array}{l}\text { Plasma adiponectin (ng/ } \\
\text { ml) }\end{array}$ & $21 \pm 6$ & $18 \pm 2$ & $26 \pm 5$ & $28 \pm 5$ & $30 \pm 7$ & $28 \pm 7$ & $24 \pm 5$ & $32 \pm 11$ \\
\hline Plasma leptin (ng/ml) & $7 \pm 2$ & $6 \pm 1$ & $67 \pm 34$ & $189 \pm 121^{*}$ & $3 \pm 1$ & $4 \pm 2$ & $6 \pm 3$ & $11 \pm 4^{* *}$ \\
\hline
\end{tabular}

in circulating neutrophils was observed in 11-mo-old ODM males fed a HFD (Figure 5b). No difference in neutrophils was observed in females at any age evaluated (data for 11-mo-old females are shown in Figure 5c). Neutrophils are short-lived leukocytes that are replenished continually by myeloid progenitor cells that reside in the bone marrow (26). To determine if ODM males fed a HFD have altered myeloid progenitor cells in the bone marrow, the cells from the femurs of OCM and ODM males were collected and quantified using standard clonogenic progenitor assays. At 6 mo of age, myeloid progenitors and total bone marrow cells were significantly increased in the ODM males fed a HFD (Figure 5d,g). In 11-mo-old males, consumption of a HFD increased myeloid progenitor cells and bone marrow cellularity for both OCM and ODM groups compared to rats fed a normal diet (Figure 5e,h). However, the most dramatic increases in myeloid progenitors and total bone marrow cells were detected in the aged ODM males fed a HFD (Figure $5 \mathrm{e}, \mathrm{h}$ ). In females, alterations in myeloid progenitors and total bone marrow cells were only observed in 11-mo-old ODM females fed a HFD (Figure 5f,i).

Previous reports demonstrate that fat-derived hormones like adiponectin and leptin regulate the growth of hematopoietic progenitors and stem cells (27-31). We assessed adiponectin and leptin levels in the serum of 11-mo-old rats. Adiponectin levels were not significantly different between OCM and ODM for males or females (Table 3). More striking were the changes in leptin concentrations. Eleven-month-old, HFD-fed ODM males had significantly increased leptin levels, consistent with the increased body weights of these rats (Table 3 ). In addition, 11-mo-old, HFD-fed ODM females had a modest increase in leptin concentrations compared to controls (Table 3). Collectively, these data link intrauterine hyperglycemia, aging, and HFD consumption to increases in leptin concentrations and altered hematopoiesis.

\section{DISCUSSION}

Our data indicate that young males exposed to hyperglycemia during the last trimester of gestation have altered insulin secretion in response to glycemic challenge. Neonatal male ODM were hypoglycemic and trended toward hyperinsulinemia, in a manner similar to human neonates exposed to GDM (4). Glucose tolerance tests in young ODM males displayed a counter-intuitive increase in glucose tolerance, similar to a prior report in rodents (18). However in contrast to the previous study, we show that the early improvements in glucose tolerance were associated with an inappropriate increase in insulin secretion compared to controls. This apparent discrepancy in findings is likely due to the timing of insulin measurement after glucose challenge. Our data demonstrate increased insulin secretion at very early timepoints $(5 \mathrm{~min})$ while no differences in insulin levels were observed at later times $(30 \mathrm{~min})(18)$. Moreover, our findings are consistent with emerging clinical data that suggest that young children of mothers with GDM exhibit improved glucose tolerance due to increased insulin secretion $(32,33)$. Additional subtle differences that exist between our data and others (18) may be due to variability in maternal hyperglycemia as tight glycemic control is challenging in the rat GDM model. Furthermore, a number of hyperglycemic dams in our study died during labor, which could have masked potential birth weight alterations detected previously. Another important point is the distinct processes for selection of offspring for crossfostering, which included only the largest pups in the prior report and was random in our study. In general, however, the findings between studies are comparable. Together these observations, in humans and rodents, support early $\beta$ cell dysfunction in offspring of diabetic mothers. While in utero, a hyperreponsive fetal $\beta$ cell would be beneficial to combat a hyperglycemic environment. However, continued insulin hypersecretion by the $\beta$ cell would be predicted to have detrimental effects, promoting increased adiposity and insulin resistance (34-36). In addition, prolonged hypersecretion of insulin may contribute to endoplasmic reticulum stress, resulting in $\beta$ cell exhaustion and/or apoptosis $(37,38)$.

To test whether external postnatal stresses potentiate the risk for ODM to develop insulin resistance, rats were fed a HFD and allowed to age. While HFD reduced glucose tolerance slightly in young (2-mo-old) OCM and ODM males, a synergistic effect of being a male ODM, aging, and consumption of a HFD yielded the highest plasma insulin levels and the most severe impairments in glucose tolerance. Increased insulin levels in the context of impaired glucose tolerance infer that the HFD-fed, aged ODM males were insulin resistant. While increased adiposity from consumption of a HFD is a wellappreciated contributor to insulin resistance (23), quantitative measurements of adiposity were not conducted in the current study; so, direct correlations with detected metabolic perturbations are not possible and would be interesting to pursue in future studies. 


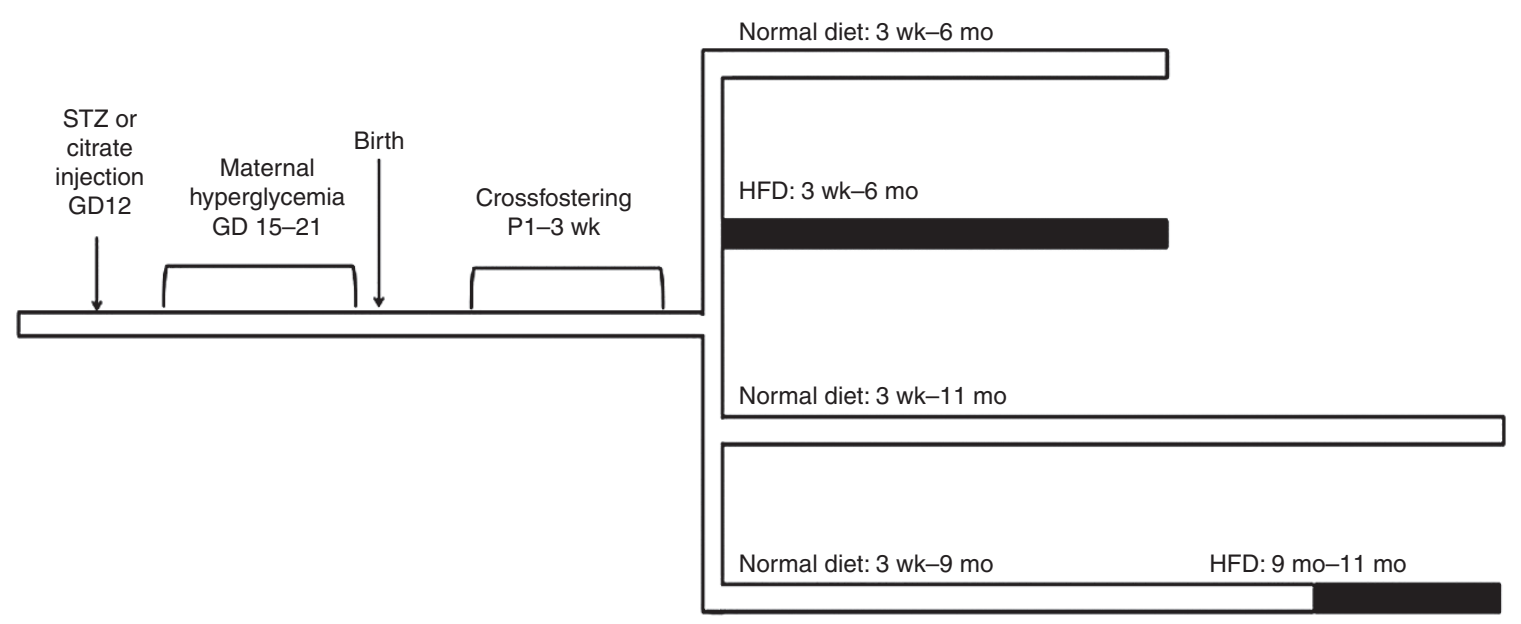

Figure 6. Schematic of the rat gestational diabetes mellitus model and experimental procedures. HFD, high-fat diet; STZ, streptozotocin.

Presumably, early $\beta$ cell dysfunction together with increased insulin resistance in male ODM enhances the lifetime risk to develop type 2 diabetes. Given these observations, it is interesting to speculate that the mechanism responsible for the early, sustained alterations in insulin secretion may be due to aberrant epigenetic regulation of key molecules involved in $\beta$ cell function and/or development. Previous studies in an intrauterine growth restriction rat model demonstrated that intrauterine growth restriction offspring had impaired epigenetic regulation of a key transcription factor, pancreatic and duodenal homeobox 1 , that regulates pancreatic development and maintains $\beta$ cell function (39). Furthermore, alterations in the regulation of pancreatic and duodenal homeobox 1 expression were linked to a decreased insulin secretory response to glucose and the development of type 2 diabetes. Whether a similar mechanism occurs in ODM is unknown, however our data suggesting early $\beta$ cell dysfunction in rat ODM prior to insulin resistance support the future interrogation of this question.

A limitation of our studies involves the possibility that STZ may cross the placenta and directly affect the fetal pancreas. Although this rat model of GDM has been used in several previous studies (16-19), direct assessment of this point has not been reported. STZ, a glucose analog, is imported by $\beta$ cells via the GLUT2 transporter (40) followed by induction of $\beta$ cell necrosis (25). Experimental rationale for use of STZ to model late gestational hyperglycemia without directly affecting the fetus includes the short half life of STZ (40) and reduced fetal levels of GLUT2 compared to adults $(41,42)$. While a possibility exists that STZ crosses the placenta, we detected no evidence of $\beta$ cell loss or reduced insulin production in neonatal ODM pups.

Significant alterations in hematopoiesis were detected in ODM males on a HFD as early as 6 mo of age (i.e., increased bone marrow cellularity and myeloid progenitors). These abnormalities intensified with age and preceded the development of neutrophilia in HFD-fed, aged ODM males. A similar, albeit delayed, pathological progression was observed in females. Neutrophilia is extremely relevant in the context of obesity-associated inflammation. Several studies establish an important role for neutrophils in the initiation and propagation of obesity-associated inflammation $(22,43,44)$. Neutrophils are mobilized from the bone marrow to initiate an inflammatory response in an acute setting or for a sustained inflammatory response in chronic diseases (45). This is achieved by neutrophils secreting cytokines, proteases, and chemokines that recruit other immune cells, especially macrophages. Adipose-associated macrophages are well appreciated as contributors to obesity-related inflammation $(24,46)$. The expansion of bone marrow myeloid progenitors and cellularity may have been facilitated by high leptin concentrations. Leptin is known to regulate hematopoiesis, and leptin receptor-positive cells are important components of the bone marrow microenvironment (29-31). Future studies that examine the interplay between alterations in adipokines, metabolism, and inflammation will promote our current understanding of the predisposition of ODM to develop components of the metabolic syndrome.

Interestingly, ODM rat females were relatively protected from the changes in glucose tolerance, insulin secretion, and hematopoiesis observed in the ODM males. Our data are consistent with previous studies in Sprague-Dawley rats that showed that females are resistant to diet-induced obesity (47). Some modest changes in weight and glucose tolerance were documented in the ODM females, especially with aging and HFD. While increased bone marrow cellularity and myeloid progenitors were observed in aged, HFD-fed females, neutrophilia was not detected up to 11 mo of age. We speculate that further aging, additional stressors, or other genetic backgrounds may be required for significant perturbations in metabolism and hematopoiesis to occur in female ODM.

In sum, these studies demonstrate metabolic and hematopoietic alterations in offspring exposed to late gestation hyperglycemia combined with aging and postnatal HFD consumption. Future studies to elucidate the molecular mechanisms involved in the evolution of these pathologic phenotypes are warranted. Given limited animal data on the effects of GDM on offspring, our data enhance current knowledge and provide the foundation for those future studies. 


\section{METHODS}

\section{In Vivo GDM Model}

All animal studies followed the guidelines for care of animals at Indiana University and were approved by Indiana University Institutional Animal Care and Use Committee. A schematic of the GDM model and postnatal exposures is shown in Figure 6. Timed pregnant SpragueDawley rats were obtained at gestational day 9 (Harlan, Indianapolis, IN). On day 12 of gestation, while under isoflurane anesthesia, animals were injected in the tail vein with either citrate buffer (control mothers) or $45 \mathrm{mg} / \mathrm{kg}$ streptozotocin (GDM mothers) (Sigma, St Louis, MO). Glucose levels were checked every morning from gestational day 15 until parturition using an Accu-Check Aviva glucose monitor (Roche, Indianapolis, IN). An average morning blood glucose level of $8.9 \mathrm{mmol} / \mathrm{l}$ $(160 \mathrm{mg} / \mathrm{dl})$ was set as the minimum for inclusion in the study. Ten percent of STZ-injected dams (3/30) had glucose values that ranged from $4.5-8 \mathrm{mmol} / \mathrm{l}(81-148 \mathrm{mg} / \mathrm{dl})$ and were excluded from the study since they were not sufficiently hyperglycemic (1). Ninety percent of STZinjected dams $(27 / 30)$ had mean glucose levels greater than $11 \mathrm{mmol} / \mathrm{l}$ $(200 \mathrm{mg} / \mathrm{dl})$. Sixty percent of dams $(18 / 30)$ received at least one injection of insulin glargine (1U, Lantus, Sanofi-Aventis, Bridgewater, NJ) or Humulin (1U, Eli Lilly, Indianapolis, IN) subcutaneously when glucose levels were above $28 \mathrm{mmol} / \mathrm{l}(500 \mathrm{mg} / \mathrm{dl})$. Pups were born spontaneously, and crossfostered to noninjected mothers in litters of 10 pups ( 5 male and 5 female) at postnatal day 1 . At $3 \mathrm{wk}$ of age, animals were weaned and fed either a normal diet (14\% kcal from fat, Teklad 7001, Harlan Laboratories, Madison, WI) or a HFD (60\% kcal from fat, TD.06414, Teklad, Harlan Laboratories) ad libitum. Animals were weighed weekly and were group housed until they reached weights of $500 \mathrm{~g}$. One cohort of animals was euthanized at 6 mo of age, at which time complete blood cell counts were performed, and plasma and bone marrow were collected (Figure 6). A separate cohort of rat offspring were allowed to consume normal diet until the age of 9 mo, when they were started on the HFD for 2 mo (Figure 6). Aged animals were euthanized at 11 mo of age at which time complete blood cell counts were performed, and plasma and bone marrow were collected.

\section{Quantitation of Pancreatic $\beta$ Cell Area}

Pancreata were isolated from control and STZ-treated dams after delivery of pups, and from neonatal male and female rats on postnatal day 1. Specimens were fixed in formalin overnight, embedded in paraffin, and sectioned by the Histology Core of the Department of Anatomy and Cell Biology at the Indiana University School of Medicine. Staining for insulin was performed (sc-9168, 1:500, Santa Cruz Biotechnology, Dallas, TX), with detection using Impress anti-rabbit horseradish peroxidase and Novared substrate (Vector Laboratories, Burlingame, CA). A Zeiss Axio Observer Z1 inverted microscope (Zeiss, Thornwood, NY) equipped with an Orca ER CCD camera (Hamamatsu Photonics, Hamamatsu City, Japan) was used to acquire digital images of the entire stained longitudinal pancreatic section. The $\beta$ cell area of at least five sections, each separated by at least $75 \mu \mathrm{m}$, from at least three animals in each group was calculated using Axio-Vision Software (Zeiss) as previously described (48).

\section{Glucose Tolerance Test}

After a 16-h fast, animals were injected with glucose ( $2 \mathrm{~g} / \mathrm{kg}$ i.p. $)$ at the ages of 4,8 , and $13 \mathrm{wk}$. Glucose levels were monitored from the tail vein using an Accu-Check Aviva glucose monitor (Roche). Plasma samples were obtained for insulin measurements in a separate cohort of animals at earlier time points. Plasma was isolated and stored at $-80^{\circ} \mathrm{C}$ until used for radioimmunoassays. Since 11 -mo-old rats on a HFD had a qualitative increase in abdominal adiposity, intravenous glucose tolerance tests were conducted under anesthesia to enhance reliability of glucose administration and decrease procedural stress for these animals. In the 11-mo-old animals, the rats were fasted and anesthetized with isoflurane before tail vein injection of glucose $(0.5 \mathrm{~g} / \mathrm{kg})$. Glucose levels were monitored from the tail vein using an Accu-Check Aviva glucose monitor.

\section{Insulin Tolerance Test}

After a 2-h fast, 8-wk-old animals were injected with insulin $(0.75 \mathrm{U} /$ kg i.p., Humulin, Eli Lilly) and tail vein blood glucose levels were measured using an Accu-Check Aviva glucose monitor (Roche).

\section{Plasma Insulin and Adipokine Measurements}

Trunk blood was collected from neonatal rats for glucose and insulin measurements. Frozen plasma samples were thawed and assayed for insulin (from glucose tolerance test in 8-wk-old and from random-fed 11-mo-old rats measured using a radioimmunoassay kit), leptin (from random-fed 11-mo-old rats using a radioimmunoassay kit) and for adiponectin (from random-fed 11-mo-old rats using an enzyme-linked immunosorbent assay kit). All assay kits were obtained from EMD Millipore, Billerica, CA. Assays were performed in duplicate, and standard curves were performed with each assay. The limit of detection for the insulin radioimmunoassay was $0.01 \mathrm{ng} / \mathrm{ml}$ for a $100 \mu \mathrm{l} \mathrm{sample.} \mathrm{Several} \mathrm{baseline} \mathrm{(time} 0$ ) values for insulin levels in the glucose tolerance test were below the limit of detection. In order to complete repeated measures ANOVA, values of limit of detection/2 were used to replace the missing values for repeated measures ANOVA.

\section{Complete Blood Cell Count}

Blood was sampled from 6- and 11-mo-old OCM and ODM in ethylenediaminetetraacetic acid-coated tubes (Sarstedt, Newton, NC), and leukocytes quantitated using a Hemavet (Drew Scientific, Waterbury, CT).

\section{Bone Marrow Progenitor Colony Assays}

To obtain bone marrow, femurs from 6- and 11-mo-old rats were flushed with Iscove's modified Dulbecco's medium (Life Technologies, Grand Island, NY) containing 20\% fetal bovine serum (Atlanta Biologicals, Flowery Branch, GA). Cells were washed and counted using a Coulter counter (Beckman Coulter, Brea, CA) to determine total femur cellularity. Cells (50,000 per $35-\mathrm{mm}$ dish) were plated in triplicate in $40 \%$ methylcellulose media (M3134, Stem Cell Technologies, Vancouver, Canada), 45\% fetal bovine serum, 0.69 $\mathrm{nmol} / \mathrm{l}$ granulocyte macrophage colony-stimulating factor (10 ng/ $\mathrm{ml}$ mouse granulocyte-macrophage colony-stimulating factor, Peprotech, Rocky Hill, NJ) and $2.7 \mathrm{nmol} / \mathrm{l}$ stem cell factor $(50 \mathrm{ng} / \mathrm{ml}$ stem cell factor; Peprotech), $2 \mathrm{U} / \mathrm{ml}$ erythropoietin (Epogen, Amgen, Thousand Oaks, CA), $78 \mu \mathrm{mol} / 1 \beta$-mercaptoethanol (Fisher Scientific, Pittsburgh, PA), and penicillin-streptomycin (Mediatech, Manassas, VA). After $7 \mathrm{~d}$ of incubation at $37^{\circ} \mathrm{C}$, total colonies were counted to determine the frequency of progenitors. Total progenitors per femur were calculated as the product of the progenitor frequency and the total cells per femur as previously described (49).

\section{Data Analysis}

Statistical analyses and graphs were created using GraphPad Prism 6 software (GraphPad Software, San Diego, CA). Data reported in the text are mean \pm SD. Statistical analyses were performed within a specific sex group as indicated in the figure legends and tables, not between males and females. Statistical significance was determined by unpaired $t$-test, or by one-way, two-way, or repeated measures ANOVA with Tukey's or Sidak's multiple comparison tests as specified in the figure legends and tables. A $\chi^{2}$ test was used to analyze frequency of maternal deaths during labor.

\section{ACKNOWLEDGMENTS}

The authors thank Shehnaz Khan for technical support on this project. We also thank Elizabeth Rybak for providing excellent administrative support.

\section{STATEMENT OF FINANCIAL SUPPORT}

This study was supported by The Riley Children's Foundation, Indianapolis, USA (L.S.H.); U.S. National Institutes of Health (Bethesda, MD), R01 DK076912 (D.C.T.), K08 DK080225 (C.E.M.), R03 DK089147 (C.E.M.), and R01 DK093954 (C.E.M.); and VA Merit Award 1101BX001733 (Washington, DC) (C.E.M.).

Disclosure: The authors declared no conflict of interest.

\section{REFERENCES}

1. American Diabetes Association. Standards of medical care in diabetes--2014. Diabetes Care 2014;37:Suppl 1:S14-80.

2. Metzger BE, Buchanan TA, Coustan DR, et al. Summary and recommendations of the Fifth International Workshop-Conference on Gestational Diabetes Mellitus. Diabetes Care 2007;30:Suppl 2:S251-60. 
3. Dabelea D, Snell-Bergeon JK, Hartsfield CL, Bischoff KJ, Hamman RF, McDuffie RS; Kaiser Permanente of Colorado GDM Screening Program. Increasing prevalence of gestational diabetes mellitus (GDM) over time and by birth cohort: Kaiser Permanente of Colorado GDM Screening Program. Diabetes Care 2005;28:579-84.

4. Weintrob N, Karp M, Hod M. Short- and long-range complications in offspring of diabetic mothers. J Diabetes Complications 1996;10:294-301.

5. Boney CM, Verma A, Tucker R, Vohr BR. Metabolic syndrome in childhood: association with birth weight, maternal obesity, and gestational diabetes mellitus. Pediatrics 2005;115:e290-6.

6. Bunt JC, Tataranni PA, Salbe AD. Intrauterine exposure to diabetes is a determinant of hemoglobin $\mathrm{A}(1) \mathrm{c}$ and systolic blood pressure in pima Indian children. J Clin Endocrinol Metab 2005;90:3225-9.

7. Cho NH, Silverman BL, Rizzo TA, Metzger BE. Correlations between the intrauterine metabolic environment and blood pressure in adolescent offspring of diabetic mothers. J Pediatr 2000;136:587-92.

8. Wright CS, Rifas-Shiman SL, Rich-Edwards JW, Taveras EM, Gillman MW, Oken E. Intrauterine exposure to gestational diabetes, child adiposity, and blood pressure. Am J Hypertens 2009;22:215-20.

9. Blondeau B, Joly B, Perret C, et al. Exposure in utero to maternal diabetes leads to glucose intolerance and high blood pressure with no major effects on lipid metabolism. Diabetes Metab 2011;37:245-51.

10. Ding GL, Wang FF, Shu J, et al. Transgenerational glucose intolerance with Igf2/H19 epigenetic alterations in mouse islet induced by intrauterine hyperglycemia. Diabetes 2012;61:1133-42.

11. Bond $\mathrm{H}$, Hamilton $\mathrm{K}$, Balment RJ, et al. Diabetes in rat pregnancy alters renal calcium and magnesium reabsorption and bone formation in adult offspring. Diabetologia 2005;48:1393-400.

12. Zhao J, Weiler HA. Long-term effects of gestational diabetes on offspring health are more pronounced in skeletal growth than body composition and glucose tolerance. Br J Nutr 2010;104:1641-9.

13. Nehiri T, Duong Van Huyen JP, Viltard M, et al. Exposure to maternal diabetes induces salt-sensitive hypertension and impairs renal function in adult rat offspring. Diabetes 2008;57:2167-75.

14. Holemans K, Gerber RT, Meurrens K, De Clerck F, Poston L, Van Assche FA. Streptozotocin diabetes in the pregnant rat induces cardiovascular dysfunction in adult offspring. Diabetologia 1999;42:81-9.

15. Gauguier D, Bihoreau MT, Picon L, Ktorza A. Insulin secretion in adult rats after intrauterine exposure to mild hyperglycemia during late gestation. Diabetes 1991;40:Suppl 2:109-14.

16. Agoudemos M, Reinking BE, Koppenhafer SL, Segar JL, Scholz TD. Programming of adult cardiovascular disease following exposure to late-gestation hyperglycemia. Neonatology 2011;100:198-205.

17. Katkhuda R, Peterson ES, Roghair RD, Norris AW, Scholz TD, Segar JL. Sex-specific programming of hypertension in offspring of late-gestation diabetic rats. Pediatr Res 2012;72:352-61.

18. Segar EM, Norris AW, Yao JR, et al. Programming of growth, insulin resistance and vascular dysfunction in offspring of late gestation diabetic rats. Clin Sci (Lond) 2009;117:129-38.

19. Zinkhan EK, Fu Q, Wang Y, et al. Maternal hyperglycemia disrupts histone 3 lysine 36 trimethylation of the IGF-1 gene. J Nutr Metab 2012;2012:930364.

20. Centers for Disease Control and Prevention. National Diabetes Fact Sheet, 2011. http://www.cdc.gov/diabetes/pubs/estimates11.html.

21. Ogden CL, Carroll MD, Kit BK, Flegal KM. Prevalence of Obesity in the United States, 2009-2010. http://www.cdc.gov/nchs/data/databriefs/db82.pdf.

22. Elgazar-Carmon V, Rudich A, Hadad N, Levy R. Neutrophils transiently infiltrate intra-abdominal fat early in the course of high-fat feeding. J Lipid Res 2008;49:1894-903.

23. Xu H, Barnes GT, Yang Q, et al. Chronic inflammation in fat plays a crucial role in the development of obesity-related insulin resistance. J Clin Invest 2003;112:1821-30.

24. Lumeng CN, Bodzin JL, Saltiel AR. Obesity induces a phenotypic switch in adipose tissue macrophage polarization. J Clin Invest 2007;117:175-84.

25. Szkudelski T. The mechanism of alloxan and streptozotocin action in B cells of the rat pancreas. Physiol Res 2001;50:537-46.

26. Borregaard N. Neutrophils, from marrow to microbes. Immunity 2010;33:657-70.
27. DiMascio L, Voermans C, Uqoezwa M, et al. Identification of adiponectin as a novel hemopoietic stem cell growth factor. J Immunol 2007;178: 3511-20.

28. Claycombe K, King LE, Fraker PJ. A role for leptin in sustaining lymphopoiesis and myelopoiesis. Proc Natl Acad Sci USA 2008;105:2017-21.

29. Bennett BD, Solar GP, Yuan JQ, Mathias J, Thomas GR, Matthews W. A role for leptin and its cognate receptor in hematopoiesis. Curr Biol 1996;6:1170-80.

30. Fantuzzi G, Faggioni R. Leptin in the regulation of immunity, inflammation, and hematopoiesis. J Leukoc Biol 2000;68:437-46.

31. Gainsford T, Willson TA, Metcalf D, et al. Leptin can induce proliferation, differentiation, and functional activation of hemopoietic cells. Proc Natl Acad Sci USA 1996;93:14564-8.

32. Bush NC, Chandler-Laney PC, Rouse DJ, Granger WM, Oster RA, Gower BA. Higher maternal gestational glucose concentration is associated with lower offspring insulin sensitivity and altered beta-cell function. J Clin Endocrinol Metab 2011;96:E803-9.

33. Chandler-Laney PC, Bush NC, Granger WM, Rouse DJ, Mancuso MS, Gower BA. Overweight status and intrauterine exposure to gestational diabetes are associated with children's metabolic health. Pediatr Obes 2012;7:44-52.

34. Mehran AE, Templeman NM, Brigidi GS, et al. Hyperinsulinemia drives diet-induced obesity independently of brain insulin production. Cell Metab 2012;16:723-37.

35. Gray SL, Donald C, Jetha A, Covey SD, Kieffer TJ. Hyperinsulinemia precedes insulin resistance in mice lacking pancreatic beta-cell leptin signaling. Endocrinology 2010;151:4178-86.

36. Shanik MH, Xu Y, Skrha J, Dankner R, Zick Y, Roth J. Insulin resistance and hyperinsulinemia: is hyperinsulinemia the cart or the horse? Diabetes Care 2008;31:Suppl 2:S262-8.

37. Fonseca SG, Urano F, Burcin M, Gromada J. Stress hypERactivation in the $\beta$-cell. Islets 2010;2:1-9.

38. Eizirik DL, Cardozo AK, Cnop M. The role for endoplasmic reticulum stress in diabetes mellitus. Endocr Rev 2008;29:42-61.

39. Park JH, Stoffers DA, Nicholls RD, Simmons RA. Development of type 2 diabetes following intrauterine growth retardation in rats is associated with progressive epigenetic silencing of Pdx1. J Clin Invest 2008;118: 2316-24.

40. Schnedl WJ, Ferber S, Johnson JH, Newgard CB. STZ transport and cytotoxicity. Specific enhancement in GLUT2-expressing cells. Diabetes 1994;43:1326-33.

41. Hathout EH, Kumagai AK, Sangkharat A, Geffner ME, Mullen Y. Absence of GLUT2 protein in near-term fetal rat pancreatic islets. Pancreas 1997;14:318-21.

42. Hughes SJ. The role of reduced glucose transporter content and glucose metabolism in the immature secretory responses of fetal rat pancreatic islets. Diabetologia 1994;37:134-40.

43. Mansuy-Aubert V, Zhou QL, Xie X, et al. Imbalance between neutrophil elastase and its inhibitor $\alpha 1$-antitrypsin in obesity alters insulin sensitivity, inflammation, and energy expenditure. Cell Metab 2013;17: $534-48$.

44. Talukdar S, Oh da Y, Bandyopadhyay G, et al. Neutrophils mediate insulin resistance in mice fed a high-fat diet through secreted elastase. Nat Med 2012;18:1407-12.

45. Mócsai A. Diverse novel functions of neutrophils in immunity, inflammation, and beyond. J Exp Med 2013;210:1283-99.

46. Weisberg SP, McCann D, Desai M, Rosenbaum M, Leibel RL, Ferrante AW Jr. Obesity is associated with macrophage accumulation in adipose tissue. J Clin Invest 2003;112:1796-808.

47. Lu Z, Wang Z, Wang X, et al. Protection from high-fat-diet-induced impaired glucose tolerance in female Sprague-Dawley rats. Gynecol Endocrinol 2009;25:464-71.

48. Sims EK, Hatanaka M, Morris DL, et al. Divergent compensatory responses to high-fat diet between C57BL6/J and C57BLKS/J inbred mouse strains. Am J Physiol Endocrinol Metab 2013;305:E1495-511.

49. Haneline LS, Broxmeyer HE, Cooper S, et al. Multiple inhibitory cytokines induce deregulated progenitor growth and apoptosis in hematopoietic cells from Fac-/- mice. Blood 1998;91:4092-8. 